\title{
Routine Health Outcome Measurement: Development, Design, and Implementation of the Hand and Wrist Cohort
}

Ruud W. Selles, Ph.D. Robbert M. Wouters, Ph.D.,

P.T.

Ralph Poelstra, M.D. Mark J. W. van der Oest, B.Sc.

Jarry T. Porsius, Ph.D.

Steven E. R. Hovius, M.D., Ph.D.

Thybout M. Moojen, M.D.,

Ph.D.

Yara van Kooij, P.T.

Pierre-Yves Pennehouat, P.T.

Rob van Huis, P.T.

Guus M. Vermeulen, M.D.,

Ph.D.

Reinier Feitz, M.D.

Harm P. Slijper, Ph.D.

For the Hand-Wrist Study

Group

Rotterdam, Hilversum, Utrecht, and

Nijmegen, The Netherlands
Summary: Routine measurement of outcome of clinical care is increasingly considered important, but implementation in practice is challenging. This article describes (1) how the authors created and implemented a routine outcome measurement cohort of patients with hand and wrist conditions and (2) how these data are used to improve the quality of care and facilitate scientific research. Starting in 2011, routine outcome measurement was implemented at all practice sites (currently 22) of a specialized treatment center for hand and wrist conditions across The Netherlands. The authors developed five "measurement tracks," including measurements administered at predetermined time points covering all hand and wrist disorders and treatments. An online system automatically distributes measurements among patients, which can be accessed by health care professionals. Using this system, the total number of yearly assigned tracks increased up to over 16,500 in 2018 , adding up to 85,000 tracks in 52,000 patients in total. All surgeons, therapists, and other staff have direct access to individual patient data and patients have access to their treatment information using a secure patient portal. The data serve as a basis for studies on, among others, comparative effectiveness, prediction modeling, and clinimetric analyses. In conclusion, the authors present the design and successful implementation of a routine outcome measurement system that was made feasible using a highly automated data collection infrastructure, tightly linked to the patient journey and the workflow of health care professionals. The system serves not only as a tool to improve care but also as a basis for scientific research studies. (Plast. Reconstr. Surg. 146: 343, 2020.)
$\mathbf{R}$ outine measurement of the outcome of clinical care is increasingly considered important in health care. It is a key aspect of value-based health care, patient-centered care, and other quality-of-care initiatives. ${ }^{1}$ For example, the Dutch government strives to have objective outcome data on 50 percent of all health care in 2022, ${ }^{2}$ and in Sweden, outcome measurements have been part of a national registry for years. ${ }^{3}$

The goals of routine outcome measurement are multiple, including improving communication

From the Departments of Plastic, Reconstructive, and Hand Surgery and Rehabilitation Medicine, Erasmus MC, University Medical Center Rotterdam; the Department of Hand and Wrist Surgery, Xpert Clinic; the Center for Hand Therapy, Handtherapie Nederland; and the Department of Plastic Surgery, Radboud University Medical Center.

Received for publication June 21, 2020; accepted February 20, 2020.

Copyright (C) 2020 by the American Society of Plastic Surgeons

DOI: $10.1097 /$ PRS.0000000000007008 and treatment guidance at the patient level, in addition to benchmarking of outcome at the level of individual clinicians or treatment centers. This benchmark information may help to establish priorities in resource allocation, and provide clinicians and managers with valuable feedback on performance. Furthermore, routine outcome measurement systems generate large data sets that can be used in scientific research. These "big data" can help provide knowledge on, for example, comparative effectiveness, predictive factors of outcome, and psychometric properties of measurement instruments.

Although routine outcome measurement has been advocated for years, implementation in clinical practice is limited because of several

Disclosure: None of the authors has a financial interest to declare in relation to the content of this article. 
challenges. These include lack of (1) consensus on which outcome measurements should be collected; (2) appropriate information technology infrastructure for data collection; (3) time and financial resources for data collection; (4) compliance of both patients and health care providers in data collection; (5) analysis and visualization tools; and (6) knowledge to improve clinical care by using the data.

In 2009, Xpert Clinic, Handtherapie Nederland, and Erasmus MC, University Medical Center Rotterdam started an initiative to collect routine outcome data in all patients with hand and wrist disorders undergoing surgical or nonsurgical treatment in their centers. This article provides an overview of this routine outcome measurement cohort by describing its design, development, and implementation. Furthermore, we describe how the accumulated data are used to improve the quality of health care and facilitate ongoing scientific research. By sharing our lessons learned, we hope to help others overcome the hurdles to implement routine outcome measurement.

\section{PATIENTS AND METHODS}

\section{Treatment Locations and Patient Population}

Routine outcome measurement was implemented in 2011 at all practice sites (currently $n=$ 22) of Xpert Clinic and Handtherapie Nederland across The Netherlands. Presently, 23 European Board-certified (Federation of European Societies for Surgery of the Hand) hand surgeons, multiple hand surgery fellows, and more than 150 hand therapists are employed within these organizations. The organizations provide nonsurgical and surgical treatment for acute and nonacute hand and wrist disorders, excluding emergency care. Patients are referred by either their general practitioner or another medical specialist. Surgical treatment is performed only in patients with an American Society of Anesthesiologists score of 1 to 2 . Table 1 shows an overview of the most common disorders and treatments.

Before any measurement or treatment, all patients are digitally asked for permission to use their data anonymously for scientific research. If a patient does not provide informed consent, the data will only be used for direct health care purposes but not for scientific analysis. Patients can always withdraw their consent. Access to all questionnaires, including the one on informed consent, is restricted through the use of a unique secret identifier provided to the individual patient by e-mail. Approval from the local medical ethical review board is obtained for each scientific study that uses the data.

\section{Measurements}

In 2010, a working group consisting of hand surgeons, hand therapists, and researchers from Xpert Clinic, Handtherapie Nederland, and Erasmus MC developed a measurement set based on existing guidelines. ${ }^{4}$ Instruments were considered if they were of direct use for clinical care, quality assessment, or treatment outcome evaluation and had proper psychometric properties. ${ }^{4}$ Measurements only relevant for scientific research or analyses of underlying abnormality (e.g., radiographic imaging or electromyography) were excluded from routine registration. All measurements were kept to a minimum to reduce the burden and optimize compliance.

The clinician-reported outcome measurements include grip and pinch strength and range of motion, whereas patient-reported outcome measurements include pain, hand function, aesthetics, return to work/daily activities, and satisfaction with the outcome. Furthermore, the Dutch Patient Reported Experience Measure is used. ${ }^{5}$

Next, we created "measurement tracks," consisting of a specific set of measurements administered at predetermined time points for each treatment or condition. We aimed to create as few measurement tracks as possible, based on similarity in the relevance of outcome domains and time points needed to capture the patients' health status. Eventually, five main measurement tracks were developed: (1) thumb disorders, (2) wrist disorders, (3) finger disorders, (4) Dupuytren's disease, and (5) compression neuropathy. The thumb, wrist, and finger tracks were further divided into a "regular" track, including shorter follow-up and fewer measurements (e.g., for trigger finger); and an "extended" track, including longer follow-up and more measurements (e.g., for thumb base surgery). For all measurement tracks, selected time points were baseline and combinations of 6 weeks and 3, 6, and 12 months after treatment (Table 2). Table 2 shows the content of each measurement track, which is reviewed and updated every 2 years. If a patient receives multiple concurrent treatments, only one track is assigned at treatment onset by the hand therapist in collaboration with the hand surgeon. To select this single track, we developed a priority rule based on the treatment that we expected, on average, to have the most impact (Table 1). Although only a single track is assigned in these cases, all concurrent treatments are registered. The same priority rule is applied when a new treatment 


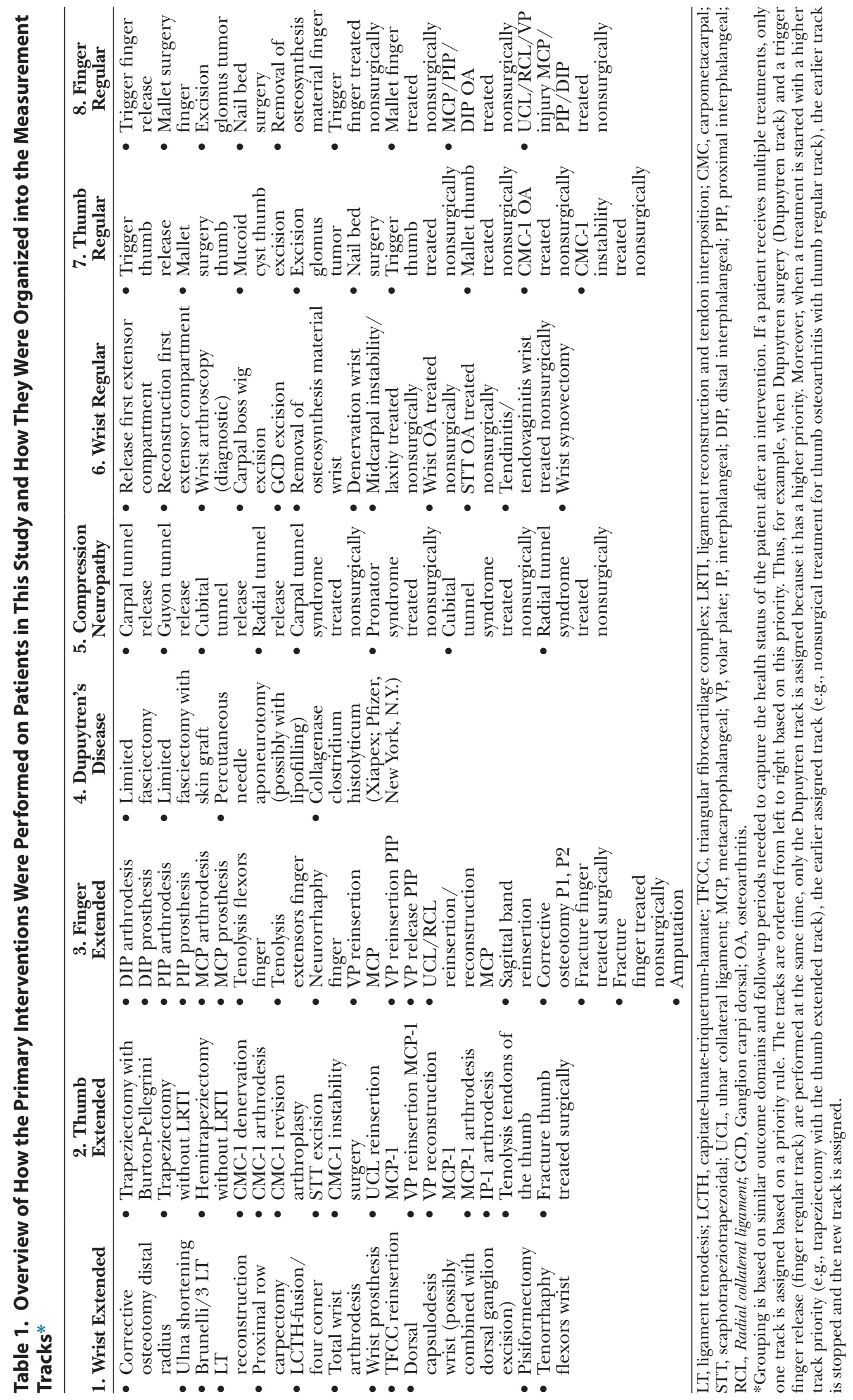


Table 2. Overview of the Predefined Tracks, Their Measurements, and Time Points*

\begin{tabular}{|c|c|c|c|c|c|}
\hline Track & Baseline & $6 \mathrm{Wk}$ & 3 Mo & $6 \mathrm{Mo}$ & 12 No \\
\hline All tracks & $\begin{array}{l}\text { - VAS: pain, } \\
\text { function, } \\
\text { satisfaction } \\
\text { - PSFS }\end{array}$ & $\begin{array}{l}\text { - VAS: pain, } \\
\text { function, } \\
\text { satisfaction } \\
\text { - PSFS } \\
\text { - Return to work } \\
\text { - Satisfaction } \\
\text { treatment result }\end{array}$ & $\begin{array}{l}\text { - VAS: pain, } \\
\text { function, } \\
\text { satisfaction } \\
\text { - PSFS } \\
\text { - Return to work } \\
\text { - Satisfaction } \\
\text { treatment result } \\
\text { - PREM }\end{array}$ & $\begin{array}{l}\text { - VAS: pain, } \\
\text { function, } \\
\text { satisfaction } \dagger \\
\text { - } \text { PSFS } \dagger \\
\text { - Return to work } \uparrow \\
\text { - Satisfaction } \\
\text { treatment result } \dagger\end{array}$ & $\begin{array}{l}\text { - VAS: pain, } \\
\text { function, } \\
\text { satisfaction } \\
\text { - PSFS } \\
\text { - Return to work } \\
\text { - Satisfaction } \\
\text { treatment result }\end{array}$ \\
\hline Thumb & $\begin{array}{l}\text { - } \mathrm{MHQ} \\
\text { - Thumb ROM } \dagger \\
\text { - Grip and pinch } \\
\text { strength } \dagger\end{array}$ & - & $\begin{array}{l}\text { - } \mathrm{MHQ} \\
\text { - Thumb ROM }+ \\
\text { - Grip and pinch } \\
\text { strength } \dagger\end{array}$ & - & $\begin{array}{l}\text { - } \mathrm{MHQ} \\
\text { - Thumb ROM } \dagger \\
\text { - Grip and pinch } \\
\text { strength } \dagger\end{array}$ \\
\hline Finger & $\begin{array}{l}\text { - } \mathrm{MHQ} \\
\text { - Finger ROM } \dagger \\
\text { - Grip strength }\end{array}$ & & $\begin{array}{l}\text { - } \mathrm{MHQ} \\
\text { - Finger ROM }+ \\
\text { - Grip strength }+\end{array}$ & - & $\begin{array}{l}\text { - } \mathrm{MHQ} \\
\text { - Finger ROM } † \\
\text { - Grip strength }\end{array}$ \\
\hline Wrist & $\begin{array}{l}\text { - PRWHE } \\
\text { - Wrist ROM } † \\
\text { - Grip strength } \dagger\end{array}$ & & $\begin{array}{l}\text { - PRWHE } \\
\text { - Wrist ROM } \dagger \\
\text { - Grip strength } \dagger\end{array}$ & - & $\begin{array}{l}\text { - PRWHE } \\
\text { - Wrist ROM } † \\
\text { - Grip strength } \dagger\end{array}$ \\
\hline $\begin{array}{l}\text { Compression } \\
\text { neuropathy }\end{array}$ & - BCTQ & & - BCTQ & - BCTQ & - \\
\hline Dupuytren & $\begin{array}{l}\text { - } \mathrm{MHQ} \\
\text { - Finger and/or } \\
\text { thumb ROM }\end{array}$ & & $\begin{array}{l}\text { - } \mathrm{MHQ} \\
\text { - Finger and/or } \\
\text { thumb ROM }\end{array}$ & - & $\begin{array}{l}\text { - } \mathrm{MHQ} \\
\text { - Finger and/or } \\
\text { thumb ROM }\end{array}$ \\
\hline
\end{tabular}

MHQ, Michigan Hand Outcome Questionnaire; VAS, visual analogue scale; VAS Function, visual analogue scale for hand function; PREM, Patient-Reported Experience Measure; PRWHE, Patient-Rated Wrist-Hand Evaluation; BCTQ, Boston Carpal Tunnel Questionnaire, ROM; range of motion; Satisfaction, satisfaction with the outcome of treatment; PSFS, Patient-Specific Functional Scale.

*This table shows the measurements performed in all tracks and the additional measurements performed in each specific track. For each type of treatment, it was decided whether patients would be assigned a regular track with a short follow-up of maximally 3 mo or an extended track with a 12-mo follow-up and more extensive measurements.

†Measurements performed only in the extended tracks for a specific time point.

starts during an already active measurement track (e.g., 3 months postoperatively) to determine whether a new track needs to be assigned.

\section{Measurement Logistics and Data Collection}

For efficient implementation of routine outcome measurement, measurement time points were aligned with the sequence of care events of typical patients (Fig. 1). For example, when a first consultation is registered in the electronic patient record, this initiates the distribution of baseline questionnaires assessing risk factors (e.g., smoking, comorbidity, and medical history) and patient expectations of the consultation and treatment. Then, during the first consultation, a hand surgeon registers the diagnosis and decides together with the patient to start either nonsurgical or surgical treatment. Based on this information, a hand therapist assigns a specific measurement track. At the same visit, the hand therapist records patient demographics (e.g., hand dominance) and clinician-reported outcome measurements and informs the patient on the treatment and future measurements. Subsequently, patient-reported outcome measurements are e-mailed to the patient. The start of nonsurgical treatment or the date of surgery determines the timing of future questionnaires or assessments. To guarantee the validity and reliability of our data, all therapists received specific training on performing the measurements.

All data are collected digitally in an online system named Pulse, which was developed using GemsTracker electronic data capture tools. ${ }^{6}$ GemsTracker is a secure, open-source, Web-based application for distribution of questionnaires and forms for clinical research and quality registration. GemsTracker uses the open-source software LimeSurvey ${ }^{7}$ for building and storing questionnaires. To ensure data safety, measurements are administered using methods similar to those in electronic patient records, including annual audits and tests, two-way authentication login, and logging and monitoring of all activity.

Because Pulse is linked to our electronic patient records, it automatically sends invitational e-mails to patients for completing questionnaires as soon as a diagnosis and treatment onset are assigned to a patient in the electronic patient record. Also, health care providers can access Pulse and see which measurements they need to complete for a specific patient.

Pulse directly calculates scores of patientreported outcome measurements and displays an overview of answered, open, and missed measures. When the same measure is administered multiple times within a track, score development over time is displayed. In the case patient-reported outcome 


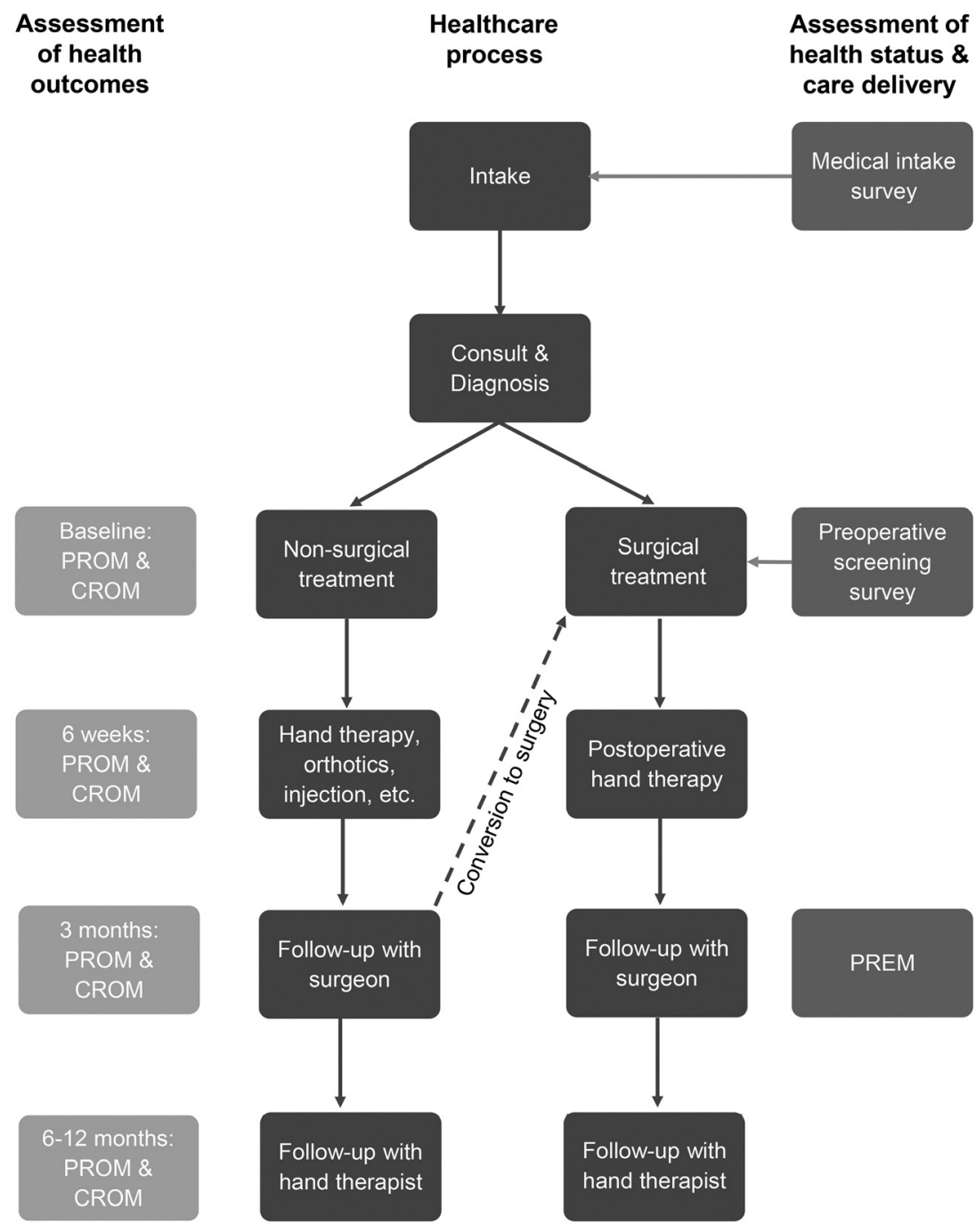

Fig. 1. Flowchart of measurement timing relative to common care paths of patients. Because the measurement system is coupled to electronic patient records with care information, measurements, and questionnaires e-mailed to patients, it can be fully automated as soon as nonsurgical or surgical treatment is entered into the system. $P R O M$, patient-reported outcome measure; $C R O M$, clinician-reported outcome measure; PREM, Patient-Reported Experience Measure.

measurements data are missing, the surgeon or therapist can request the patient to complete the missing questionnaires, but treatment can also continue without this information.

\section{RESULTS}

\section{Collected Data}

Figure 2 shows the number of tracks assigned to patients over the years. The total number of yearly assigned tracks increased up to over 16,300 in 2018, adding up to a total of 85,000 tracks in 52,000 patients. The increase in the track numbers reflects the growth in treatment volume and the opening of new centers. The regular tracks, which include nonsurgical treatments (e.g., orthotics, exercise therapy, injections) and minor surgical interventions (e.g., trigger finger release), were more often assigned than extended tracks, which include more invasive surgery. Table 3 shows that the Michigan Hand Outcomes Questionnaire, Patient-Rated Wrist/Hand Evaluation, and our Patient-Reported Experience Measure are the most time-consuming measures, with a median of 3 to 4 minutes to complete. These completion times are lower than initially reported; for example, the Michigan Hand Outcomes Questionnaire is reported to take approximately 15 minutes to complete according to its developers. ${ }^{8}$ 


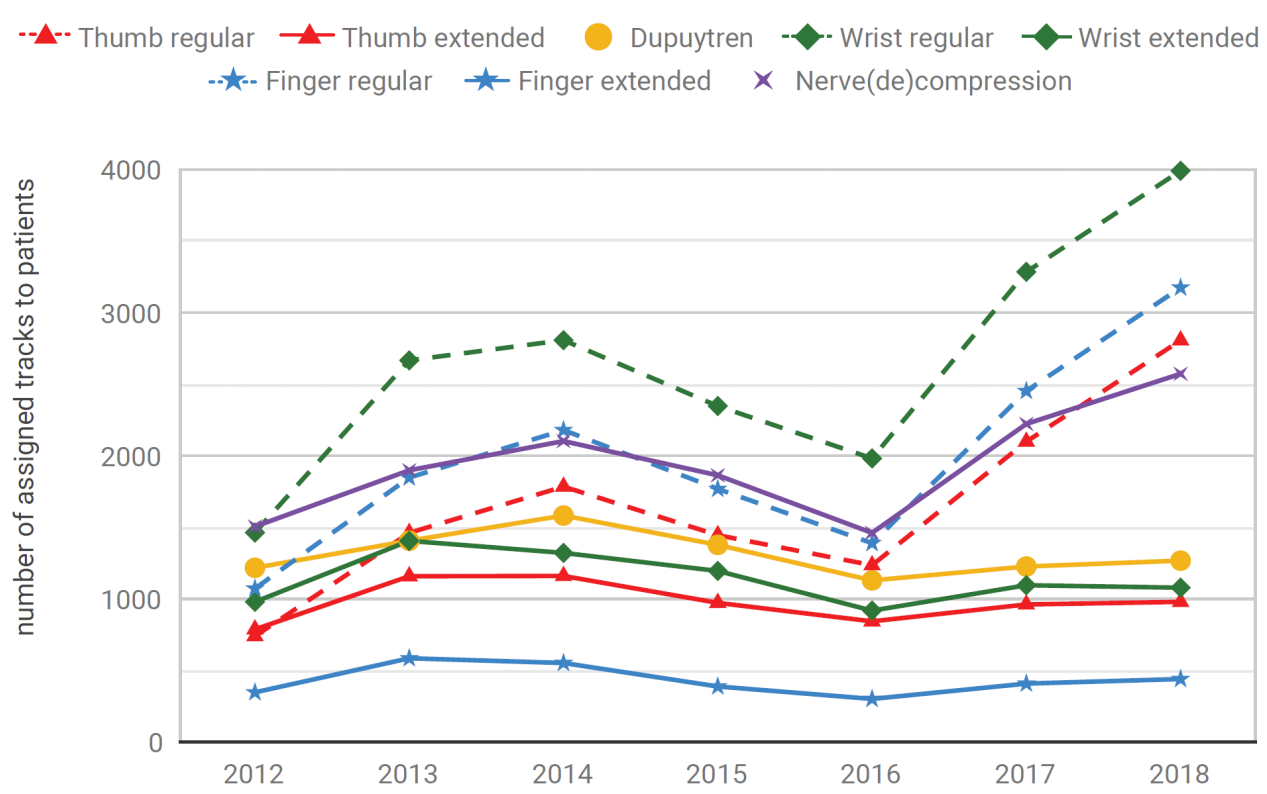

Fig. 2. The number of yearly activated measurement tracks. Dashed lines indicate the regular tracks; solid lines indicate the extended tracks. Note that more than one measurement track can be assigned to a patient, for example, when a new treatment track (e.g., surgery) is initiated after an initial treatment track failed to obtain sufficient relief of symptoms (e.g., an injection or hand therapy). The decrease in track assignment in 2015 and 2016 was attributable to organizational problems leading to a significant number of patients where a measurement track was not assigned at the start of treatment. However, as can be seen, this improved by 2017.

Patient compliance for completing questionnaires was highest at baseline. For example, for pain, hand function, and satisfaction questionnaires, compliance was 73 percent at baseline and decreased to 62 percent at 12 months (Fig. 3, above). Compliance in extended tracks was 8 percent higher at baseline and 14 percent higher at 3 months compared to regular tracks. Compliance also decreased at follow-up for clinicianreported outcome measurements (Fig. 3, below); at baseline, 90 percent of measurement forms were completed, whereas at 3 and 12 months, these

Table 3. Total Number of Patient Questionnaires across All Tracks and the Median Time to Complete the Questionnaires from 2011 to 2018

\begin{tabular}{lcc}
\hline Questionnaire & $\begin{array}{c}\text { No. of } \\
\text { Completed } \\
\text { Questionnaires }\end{array}$ & $\begin{array}{c}\text { Median } \\
\text { Time to Complete } \\
\text { (min:sec) }\end{array}$ \\
\hline MHQ & 49,925 & $4: 15$ \\
PRWHE & 28,784 & $3: 43$ \\
BCTQ & 17,680 & $1: 54$ \\
Return to work & 40,998 & $0: 39$ \\
Satisfaction with result & 81,534 & $0: 14$ \\
VAS pain and function & 135,074 & $0: 33$ \\
PREM & 25,407 & $4: 17$ \\
\hline
\end{tabular}

MHQ, Michigan Hand Outcome Questionnaire; PRWHE, PatientRated Wrist-Hand Evaluation; BCTQ, Boston Carpal Tunnel Questionnaire; VAS, visual analogue scale; PREM, Patient-Reported Experience Measure. numbers decreased to 50 percent and 38 percent, respectively.

\section{Using Outcome Data in Clinical Practice}

From the start in 2011, all surgeons, therapists, and staff had direct access to all scores of individual patients and their development over time. Thus, for example, hand therapists use the measurements to evaluate treatment progress and set new treatment goals. Also, we introduced an integrated secure patient portal (Fig. 4) to allow patients to access their treatment information. Within this portal, patients can complete their questionnaires and see their progress over time. Based on the assigned treatment, patient-specific treatment information is provided (e.g., diseasespecific instructional videos on postoperative exercises). In 2018, approximately 3100 patients logged into their patient portal each month.

From 2017 onward, we show individual patient outcomes relative to the average outcome from previous patients. For example, patients can see their pain score over time relative to mean scores of previous patients undergoing the same treatment (Fig. 5). Moreover, we introduced a physician dashboard, where physician-specific outcomes for more than 100 treatments are compared to the average of all other physicians. 
Volume 146, Number 2 • The Hand-Wrist Study Group Cohort
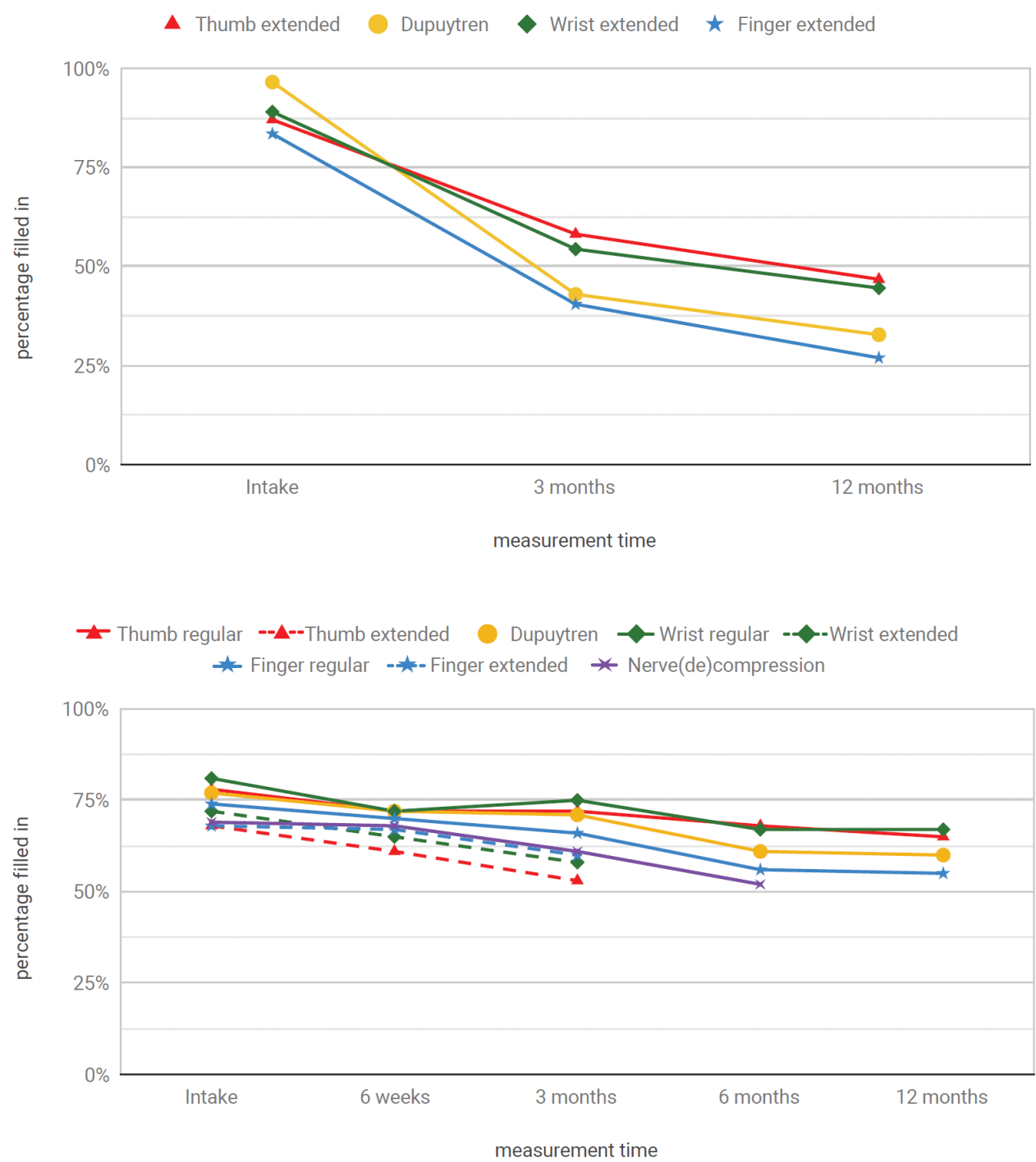

Fig. 3. (Above) Compliance of hand therapists filling in the clinician-reported outcome measurements, such as goniometry and grip strength. There are differences in compliance between measurement tracks, but the most important factor is the duration of the follow-up. (Below) Compliance of patients completing the patient-reported outcome measurements, illustrated using the compliance on the visual analogue scale for pain, hand function, and satisfaction.

\section{Scientific Research with the Collected Data}

Although our data collection system was designed primarily to improve and monitor the quality of health care of our patients, the system also constitutes a cohort of high-quality data suitable for scientific research: the Hand-Wrist Study Group Cohort.

\section{Comparative Effectiveness and Prediction Modeling}

Our first published studies ${ }^{9-13}$ focused on comparative effectiveness. In these studies, variation in daily clinical practice is used to compare different treatments (e.g., when different surgeons prefer different treatments in the same patient population). To correct for baseline differences between treatment groups, we use propensity score matching and mixed models. For example, we showed that collagenase clostridium histolyticum in Dupuytren's disease was not significantly different from limited fasciectomy in reducing metacarpophalangeal joint contractures in short-term outcome, whereas proximal interphalangeal joint contractures showed slightly better reduction following limited fasciectomy. ${ }^{3}$ Furthermore, we demonstrated that exercise therapy in addition to an orthosis reduces pain more 


Personal Page
My organization
My treatment
My results
Client instruction
My consults
My surveys
My data
My work
Frequently asked
questions
Your opinion

\begin{tabular}{l|l|l} 
Before the consult & Before the treatment After the treatment After the checkup
\end{tabular}

The carpal tunnel is located at the level of the transition from the forearm to the hand and forms a kind of 'hatch'. The tunnel is formed by eight hand bones in the shape of a " $U$ ". A sturdy band of connective tissue (transverse carpal ligament of the wrist) runs between the legs of the ' $U$ ', forming the carpal tunnel. A total of 9 tendons and 1 nerve run through this tunnel. The tendons are the spurs of muscles that are located in the forearm and allow movement of the fingers and thumb. $\gg$ Read on

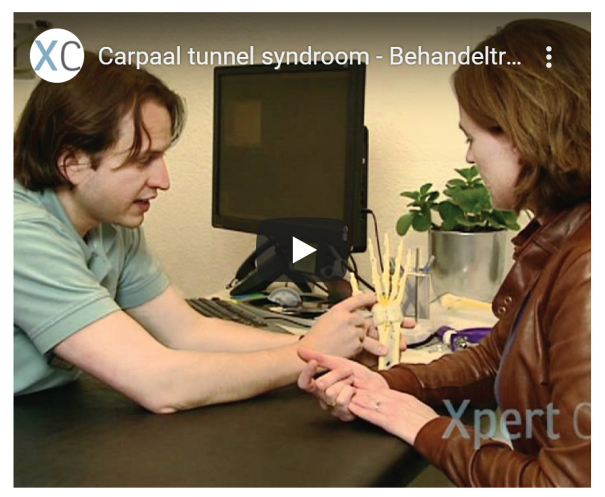

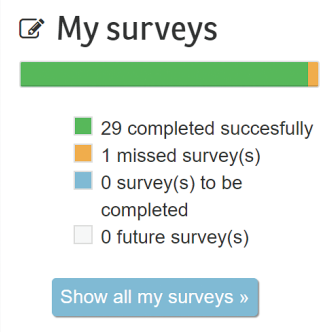

My consults

You have no planned consults. Show all my consults $»$

Fig. 4. Screenshot of the personalized patient portal, where patients can learn about the treatment, health care process, expected outcomes, and exercises and can also complete the required questionnaires. As soon as a measurement track is assigned to a patient, disease-specific information is provided.

compared to an orthosis only in patients with thumb base osteoarthritis ${ }^{13}$ and that, following a thumb carpometacarpal resection arthroplasty, shorter immobilization is noninferior compared to more prolonged immobilization. ${ }^{10}$ In addition to comparative effectiveness, we use our data to develop and validate prognostic and clinical prediction models that allow outcome prediction of individual patients, for example, on the outcome of nonsurgical treatment for thumb base osteoarthritis, ${ }^{13-16}$ surgical treatment of primary or recurrent carpal tunnel syndrome, ${ }^{17-19}$ and surgery in Dupuytren's contracture. ${ }^{20,21}$

\section{Health Care Context and Treatment Outcomes}

We also study how outcomes are influenced not only by treatment but also by the process of care delivery and patient experiences. More specifically, we consistently found positive associations between patient experiences on care delivery and improvement in patient-reported outcome measures following surgical treatments. ${ }^{5,22,23}$ The strongest associations were found for positive experiences with the communication of the surgeon and providing treatment information, which is in line with other studies. ${ }^{5,22,23}$

\section{Clinimetric Studies}

The collected data also allow evaluating the psychometric measurement properties. For example, in patients with Dupuytren's contracture, we reported that the Patient-Specific Functional Scale is more responsive than the more generic and standardized Michigan Hand Outcomes Questionnaire, despite being much shorter to fill in. ${ }^{24}$ In addition, we developed decision tree-based versions of the Patient-Rated Wrist/Hand Evaluation $^{25}$ and the Boston Carpal Tunnel Questionnaire ${ }^{26}$ to reduce the number of items needed to calculate the total score from 15 and 18 to six for both patient-reported outcome measures, without loss of information (see http://handquestionnaires.org).

\section{DISCUSSION}

We introduce the design, development, and implementation of a routine outcome measurement system in hand and wrist care, describing how our data are collected and used for improving clinical care and performing scientific research. The system was made feasible by using a highly automated data collection infrastructure, tightly linked to the patient journey and the workflow of health care professionals. With this article, we 


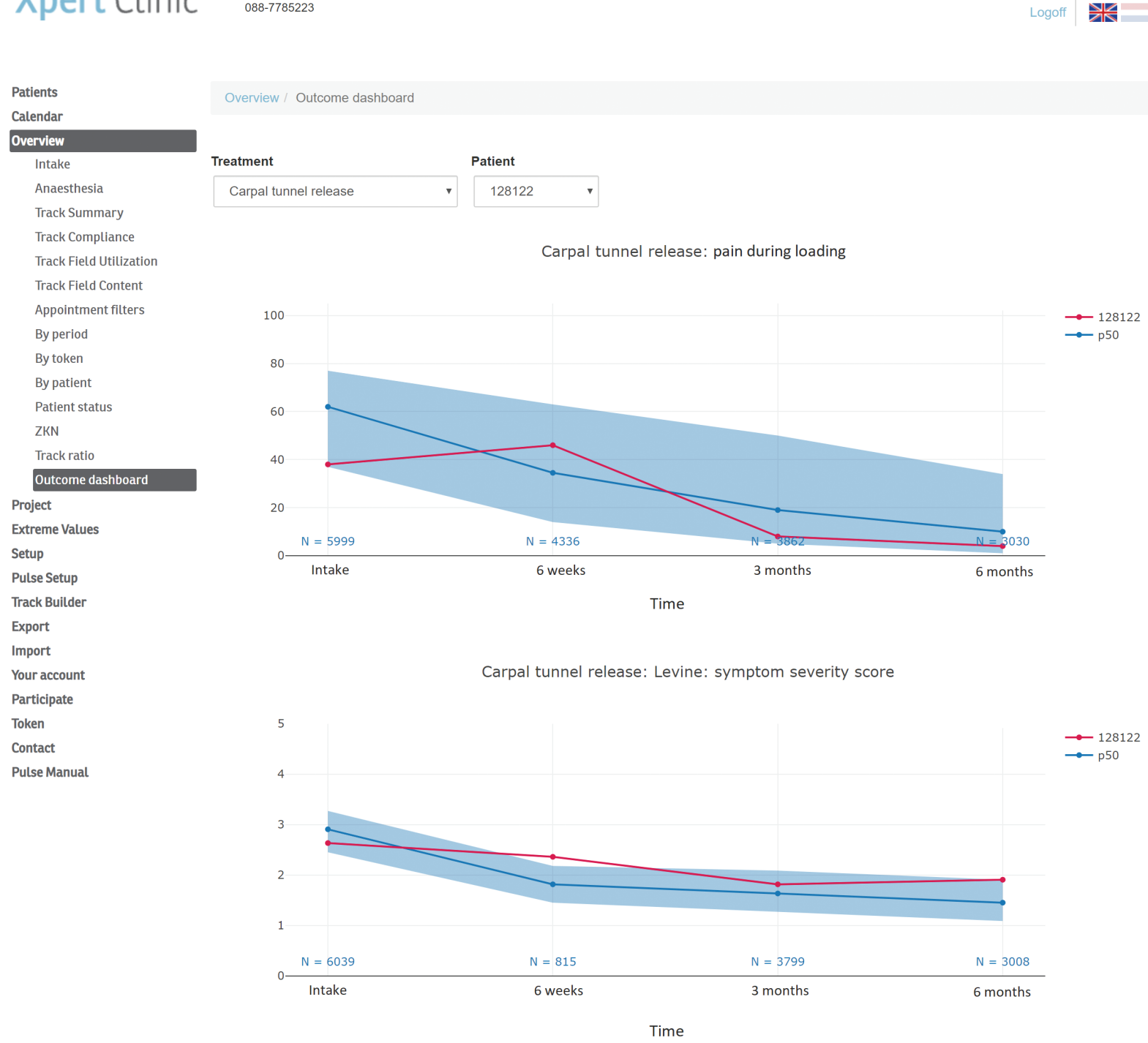

Fig. 5. Screenshot of a physician dashboard, showing the individual patient's outcome (magenta line) compared to the "average patient's" outcomes (blue line, p50; and blue area, p25 to p75) after a carpal tunnel release. The data shown can be modified by the user, who can select a treatment, a treatment location, and a surgeon. These outcomes will then be plotted compared to the outcomes of all other surgeons at all treatment locations for that treatment. (Screenshot published with permission from Xpert Clinic.)

intend to share our experiences in designing such a system and our lessons learned, and to describe the remaining challenges.

The design and implementation of our routine outcome measurement system were facilitated by the specific expertise of the collaborating parties. The Erasmus MC, as a large academic center, contributes scientific knowledge, and Xpert Clinic, as a highly specialized hand and wrist clinic, can quickly innovate and integrate the measurements in their workflow. By developing dedicated software, ${ }^{6}$ we could customize the data collection to our specific needs and implement changes efficiently.
Ensuring high compliance of both patients and clinicians remains a challenge, as in all outcome measurement systems. ${ }^{27}$ We took several measures to optimize compliance. A first step was to minimize the measurement burden and allow direct measurement feedback to both patients and clinicians. A second step was to improve data integration during consultations and therapy. For instance, instead of asking for limitations in daily life during a patient's first visit, clinicians can now see this information beforehand and can discuss these issues directly. As a third step, we visualize individual outcomes relative to other patients, 
which provides a reference for both patient and clinician to discuss treatment outcomes. At present, we present outcomes as group means plus confidence intervals at the level of specific treatments (e.g., a trapeziectomy) but this can be further personalized to individuals (e.g., a 70-year-old woman with a baseline Michigan Hand Outcomes Questionnaire score of 50). Thus, in the future, we plan to extend this and present individualized outcome predictions based on existing data.

Although clinicians value outcome information, more research is needed on how to efficiently use outcome data to improve quality of care and maintain practical feasibility. Presently, it remains challenging for clinicians to actually use the data in daily practice, for a variety of reasons, such as lack of time or inexperience in how to use the data in daily clinical practice. Another concern is that a multitude of factors can influence expected outcomes for an individual patient that need to be taken into account when discussing the expected outcome with a patient. Therefore, we are presently developing models that can predict outcome for individual patients. Our current efforts are focused on the implementation of these models in daily clinical practice so that they can be used in real time during consultation. In addition, in the future, we plan to link outcome data with the cost of treatment as recorded in the electronic health care record, providing insight into the quality of care from a value-based health care perspective.

We found that efficient data acquisition software allows outcome recording with a relatively small time investment per patient. Furthermore, at present, the main costs include software development and maintenance (approximately 2 to 3 full-time equivalents throughout the last years for all participating treatment centers) and the efforts of staff, management, and researchers to design the system. By making the GemsTracker software open-source and describing our procedures in detail, we intend to lower the costs for new centers to develop a similar system. However, despite our successful implementation, reimbursement by health care insurance companies for outcome measurement remains unusual, despite the wish of insurance companies and the government to collect outcome data. Thus, further collaboration between health care providers, scientists, insurance companies, and governments is needed, because these investments are currently being made by health care organizations themselves.

When comparing the Hand-Wrist Study Group cohort with other large cohorts and related initiatives, there are significant similarities and differences. For example, registries such as the Swedish hand registry ${ }^{28}$ have larger patient numbers but less detailed information. Other commonly used cohorts consist of administrative or claim data on the hospital, regional, or national level. ${ }^{29-32}$ To our knowledge, the present cohort is unique within the field of hand and wrist disorders because it contains a large number of patients with relatively detailed data, covering both outcomes, treatment information, and patient characteristics. A limitation, however, is that this cohort is not representative of all hand and wrist patients in The Netherlands, for example, because complex trauma patients and patients with more severe comorbidities may be treated more often elsewhere. Also, if patients seek treatment elsewhere, no follow-up is available.

For all clinical (e.g., quality evaluation and benchmarking) and scientific analysis, missing data are always an important issue. In several of our research articles, we have performed extensive missing data analysis and have consistently found that our data can be qualified as missing completely at random. ${ }^{33-36}$ In the literature, many statistical analyses and simulation articles have indicated that either multiple imputation techniques or analyses that account for missing data are superior to complete case analyses. ${ }^{33-37}$ However, we noticed that such techniques are counterintuitive to many readers. Consequently, we have frequently been asked by journal reviewers to report complete cases, despite literature advising otherwise.

Because measuring outcomes is central in value-based health care, ${ }^{1}$ it would be of great value if more health care providers in hand and wrist care would routinely measure outcomes. Although there have been several consensus initiatives on outcome sets, ${ }^{28,38-41}$ none has led to widespread implementation. We hope that our example of routine outcome measurement implementation and the development of the hand and wrist standard set by the International Consortium for Health Outcome Measurement ${ }^{42}$ will lead to a common ground for more widespread comparisons of outcomes.

Ruud W. Selles, Ph.D.
Department of Rehabilitation Medicine
Department of Plastic and Reconstructive Surgery and
Hand Surgery
Erasmus MC, University Medical Center Rotterdam
P.O. Box 2040
3000 CA Rotterdam, The Netherlands
r.selles@erasmusmc.nl
Twitter: @ruudselles

Ruud W. Selles, Ph.D. . 


\section{ACKNOWLEDGMENTS}

The authors thank all patients who completed questionnaires as part of their clinical care and agreed for their data to be used in scientific studies. In addition, they would like to acknowledge the members of the HandWrist Study Group, health care providers and personnel of Xpert Clinic, Handtherapie Nederland, and Equipe Zorgbedrijuen, for facilitating the routine outcome measurements that are the basis for this article.

\section{REFERENCES}

1. Porter ME. What is value in health care? $N$ Engl J Med. 2010;363:2477-2481.

2. Dutch National Health Care Institute. Meer patiëntregie door meer uitkomstinformatie in 2022. Available at: https:/ / www.zorginstituutnederland.nl/werkagenda/publicaties/ rapport/2018/06/28/rapport-meer-patientregie-doormeer-uitkomstinformatie-in-2022. Accessed June 8, 2020.

3. Nilsson E, Orwelius L, Kristenson M. Patient-reported outcomes in the Swedish National Quality Registers. I Intern Med. 2016;279:141-153.

4. American Society of Hand Therapists. Clinical Assessment Recommendations. 3rd ed. Mount Laurel, NJ: American Society of Hand Therapists; 2015.

5. Poelstra R, Selles RW, Slijper HP, et al.; Hand-Wrist Study Group. Better patients' treatment experiences are associated with better postoperative results in Dupuytren's disease. J Hand Surg Eur Vol. 2018;43:848-854.

6. GemsTracker; Erasmus MC; Equipe Zorgbedrijven. GemsTrakcer, latest release at 2017, version 1.8.2, open source (new BSD licence). Available at: https://gemstracker. org. Accessed June 8, 2020.

7. LimeSurvey GmbH. LimeSurvey. Available at: https://www. limesurvey.org/. Accessed June 8, 2020.

8. Michigan Hand outcomes Questionnaire (MHQ). Available at: http://mhq.lab.medicine.umich.edu/home. Accessed June 8, 2020.

9. Zhou C, Hovius SE, Slijper HP, et al. Collagenase clostridium histolyticum versus limited fasciectomy for Dupuytren's contracture: Outcomes from a multicenter propensity score matched study. Plast Reconstr Surg. 2015;136:87-97.

10. Tsehaie J, Wouters RM, Feitz R, Slijper HP, Hovius SER, Selles RW; Hand-Wrist Study Group. Shorter vs longer immobilization after surgery for thumb carpometacarpal osteoarthritis: A propensity score-matched study. Arch Phys Med Rehabil. 2019;100:2022-2031.e1.

11. Zhou C, Selles RW, Slijper HP, et al. Comparative effectiveness of percutaneous needle aponeurotomy and limited fasciectomy for Dupuytren's contracture: A multicenter observational study. Plast Reconstr Surg. 2016;138:837-846.

12. Zhou C, Hovius SER, Pieters AJ, Slijper HP, Feitz R, Selles RW. Comparative effectiveness of needle aponeurotomy and collagenase injection for Dupuytren's contracture: A multicenter study. Plast Reconstr Surg Glob Open 2017;5:e1425.

13. Wouters RM, Tsehaie J, Slijper HP, Hovius SER, Feitz R, Selles RW; Hand-Wrist Study Group. Exercise therapy in addition to an orthosis reduces pain more than an orthosis alone in patients with thumb base osteoarthritis: A propensity score matching study. Arch Phys Med Rehabil. 2019;100:1050-1060.

14. Tsehaie J, Porsius JT, Rizopoulos D, et al. Response to conservative treatment for thumb carpometacarpal osteoarthritis is associated with conversion to surgery: A prospective cohort study. Phys Ther. 2019;99:570-576.
15. Tsehaie J, Spekreijse KR, Wouters RM, et al. Outcome of a hand orthosis and hand therapy for carpometacarpal osteoarthritis in daily practice: A prospective cohort study. J Hand Surg Am. 2018;43:1000-1009.e1.

16. Tsehaie J, Spekreijse KR, Wouters RM, et al.; Hand-Wrist Study Group. Predicting outcome after hand orthosis and hand therapy for thumb carpometacarpal osteoarthritis: A prospective study. Arch Phys Med Rehabil. 2019;100:844-850.

17. Jansen MC, Evers S, Slijper HP, et al. Predicting clinical outcome after surgical treatment in patients with carpal tunnel syndrome. J Hand Surg Am. 2018;43:1098-1106.e1.

18. Evers S, Jansen MC, Slijper HP, et al. Hand surgeons performing more open carpal tunnel releases do not show better patient outcomes. Plast Reconstr Surg. 2018;141:1439-1446.

19. Sun PO, Selles RW, Jansen MC, Slijper HP, Ulrich DJO, Walbeehm ET. Recurrent and persistent carpal tunnel syndrome: Predicting clinical outcome of revision surgery. $J$ Neurosurg. 2019:1-9. Epublished ahead of print.

20. Zhou C, Ceyisakar IE, Hovius SER, et al. Surgeon volume and the outcomes of Dupuytren's surgery: Results from a Dutch multicenter study. Plast Reconstr Surg. 2018;142:125-134.

21. Zhou C, Hovius SE, Slijper HP, et al. Predictors of patient satisfaction with hand function after fasciectomy for Dupuytren's contracture. Plast Reconstr Surg. 2016;138:649-655.

22. Tsehaie J, van der Oest MJW, Poelstra R, et al. Trapeziometacarpal osteoarthritis: Positive experience with treatment is associated with better surgical outcome. J Hand Surg Eur Vol. 2019;44:714-721.

23. Festen-Schrier VJMM, Poelstra R, Selles RW, et al. Better patient-reported experiences with health care are associated with improved clinical outcome after carpal tunnel release surgery. Plast Reconstr Surg. 2019;143:1677-1684.

24. van Kooij Y, Poelstra R, Porsius JT, Slijper HP, Warwick D, Selles RW. The Patient Specific Functional Scale in Dupuytren's disease; a more responsive outcome measure than standardized PROMS? Paper presented at: 14th IFSSH11th IFSHT; June 17-21, 2019; Berlin, Germany.

25. van der Oest MJW, Porsius JT, MacDermid JC, Slijper HP, Selles RW. Item reduction of the patient-rated wrist evaluation using decision tree modelling. Disabil Rehabil. 2019:1-8. Epublished ahead of print.

26. Jansen MC, van der Oest MJ, Slijper HP, Porsius JT, Selles RW. Item reduction of the Boston Carpal Tunnel Questionnaire using decision tree modeling. Arch Phys Med Rehabil. 2019;100:2308-2313.

27. Edwards PJ, Roberts I, Clarke MJ, et al. Methods to increase response to postal and electronic questionnaires. Cochrane Database Syst Rev. 2009;3:MR000008.

28. Arner M. Developing a national quality registry for hand surgery: Challenges and opportunities. EFORT Open Rev. 2016;1:100-106.

29. de Putter CE, Selles RW, Polinder S, Panneman MJ, Hovius SE, van Beeck EF. Economic impact of hand and wrist injuries: Health-care costs and productivity costs in a populationbased study. J Bone Joint Surg Am. 2012;94:e56.

30. Sears ED, Swiatek PR, Hou H, Chung KC. The influence of insurance type on management of carpal tunnel syndrome: An analysis of nationwide practice trends. Plast Reconstr Surg. 2016;138:1041-1049.

31. Manley OWG, Wormald JCR, Furniss D. The changing shape of hand trauma: An analysis of hospital episode statistics in England. J Hand Surg Eur Vol. 2019;44:532-536.

32. Craig RS, Lane JCE, Carr AJ, Furniss D, Collins GS, Rees JL. Serious adverse events and lifetime risk of reoperation after elective shoulder replacement: Population based cohort study using hospital episode statistics for England. BMJ2019;364:1298. 
33. de Groot JA, Janssen KJ, Zwinderman AH, Bossuyt PM, Reitsma JB, Moons KG. Correcting for partial verification bias: A comparison of methods. Ann Epidemiol. 2011;21:139-148.

34. Little RJA. A test of missing completely at random for multivariate data with missing values. JAm Stat Assoc. 1988;83:1198-1202.

35. Little RJ, D'Agostino R, Cohen ML, et al. The prevention and treatment of missing data in clinical trials. N Engl J Med. 2012;367:1355-1360.

36. Zeger SL, Liang KY. Longitudinal data analysis for discrete and continuous outcomes. Biometrics 1986;42:121-130.

37. Martín-Merino E, Calderón-Larrañaga A, Hawley S, et al. The impact of different strategies to handle missing data on both precision and bias in a drug safety study: A multidatabase multinational population-based cohort study. Clin Epidemiol. 2018;10:643-654.

38. van de Ven-Stevens LA, Graff MJ, Selles RW, et al. Instruments for assessment of impairments and activity limitations in patients with hand conditions: A European Delphi study. $J$ Rehabil Med. 2015;47:948-956.
39. Goldhahn J, Beaton D, Ladd A, Macdermid J, Hoang-Kim A; Distal Radius Working Group of the International Society for Fracture Repair (ISFR); International Osteoporosis Foundation (IOF). Recommendation for measuring clinical outcome in distal radius fractures: A core set of domains for standardized reporting in clinical practice and research. Arch Orthop Trauma Surg. 2014;134:197-205.

40. Waljee JF, Ladd A, MacDermid JC, Rozental TD, Wolfe SW; Distal Radius Outcomes Consortium. A unified approach to outcomes assessment for distal radius fractures. J Hand Surg Am. 2016;41:565-573.

41. Wittoek R, Kroon FPB, Kundakci B, et al. Report from the Hand Osteoarthritis Working Group at OMERACT 2018: Update on core instrument set development. J Rheumatol. 2019;46:1183-1187.

42. International Consortium for Health Outcome Measurement (website). Available at: https://www.ichom.org/. Accessed June 8, 2020. 